\title{
Estimation of the maximum contraction velocity of the urinary bladder from pressure and flow throughout micturition
}

\author{
R. van Mastrigt \\ Departments of Urology and Biomedical Physics and Technology, Erasmus University, Rotterdam, The Netherlands
}

Accepted: May 19, 1989

Summary. The contractility of the urinary bladder can be adequately described in terms of the parameters $\mathrm{P}_{0}$ (isometric pressure) and $\mathrm{v}_{\max }$ (maximum contraction velocity). In about $12 \%$ of urodynamic evaluations of patients these clinically relevant parameters can be calculated from pressure and flow rate as measured during micturition. A method was developed of estimating $\mathrm{v}_{\max }$ for any micturition from these signals. The properties of this estimated contractility parameter were clinically tested and are discussed.

Key words: Contractility - Urinary bladder - Maximum contraction velocity - Contraction velocity - Velocity Hill equation - Smooth muscle

The contractility of a muscle is modeled by the Hill equation in the form of a hyperbolic relation between contraction velocity and force exerted by the muscle [6]. For a hollow spherical muscle such as the urinary bladder a similar relation can be derived between the circumferential shortening velocity and the pressure of the fluid within the muscle lumen $[7,2]$. Such a relation defines the shortening or emptying behaviour of the muscle in terms of three parameters, which generally depend on and therefore vary with muscle length and degree of stimulation. The standard form of the Hill equation:

$(P+a)(v+b)=\left(P_{0}+a\right) b$

can be rewritten as follows:

$$
\begin{aligned}
& \left(\mathrm{P} / \mathrm{P}_{0}+\mathrm{a} / \mathrm{P}_{0}\right)\left(\mathrm{v}+\left(\mathrm{a} / \mathrm{P}_{0}\right) \cdot\left(\mathrm{v}_{\max }\right)\right. \\
& =\left(1+\left(\mathrm{a} / \mathrm{P}_{0}\right)\right) \cdot \mathrm{a} / \mathrm{P}_{0} \cdot \mathrm{v}_{\max }
\end{aligned}
$$

so that the three parameters are $\mathrm{P}_{0}, \mathrm{v}_{\max }$, and $\mathrm{a} / \mathrm{P}_{0}$. $a / P_{0}$ is a parameter characterizing the curvature of the hyperbola intersecting the pressure and velocity axes at $\mathbf{P}_{0}$ and $v_{\max }$. $\mathrm{a} / \mathrm{P}_{0}$ (or $\mathrm{a} / \mathrm{F}_{0}$ for muscle strips) is generally found to be a constant approximately equal to 0.25 for urinary bladder muscle and most other types of muscle [5, 13,7]. Inserting this value into Eq. 2 yields the two-parameter equation:

$\left(\mathrm{P}+\mathrm{P}_{0} / 4\right)\left(\mathrm{v}+\mathrm{v}_{\max } / 4\right)=5 \cdot \mathrm{P}_{0} \cdot \mathrm{v}_{\max } / 16$

From measurements of detrusor pressure and flow rate during micturition, a plot of the circumferential shortening velocity as a function of detrusor pressure can be made [2]. Generally such a plot shows the complicated interaction of changes in contractility (due to varying stimulation and shortening of the muscle) and urethral resistance (due to varying stimulation). In a limited number of measurements however rapid changes in the urethral resistance relation occur and part of the hyperbolic equation Eq. 3 is shown. In such cases the parameters $P_{0}$ and $v_{\max }$ can be estimated by fitting a hyperbola to that part of the P-v plot (see Results, Figs. 1 and 2). The fitting requires both a translation and a rotation of the fitting curve, see Fig. 3. This procedure for calculating the parameters $P_{0}$ and $v_{\max }$ can only be applied to a very limited number of urodynamic measurements (Eq. 11). In the present study, two methods of estimating the contractility parameter $\mathrm{v}_{\max }$ from any pressure-flow study are investigated.

The first method is based on an experimentally determined relation between the degree of rotation of the hyperbola with the maximum flow in the pressure-flow measurement. An estimate of $\mathrm{v}_{\max }$ can be obtained by translating a hyperbola with preset rotation (based on the maximum flow) until it just touches the highest point in the presentation of the data. Conceptually this is equivalent to using a constant normalized Hill equation [1]. The second method involves the estimation of $\mathrm{P}_{0}$ from conventional urodynamic parameters, and calculating a hyperbola through this point and the point with maximum velocity in the pressure-velocity plot. The derivation and reliability of these methods for estimating the maximum contraction velocity of the urinary bladder and some indications of their usefulness as contractility parameters are discussed. 


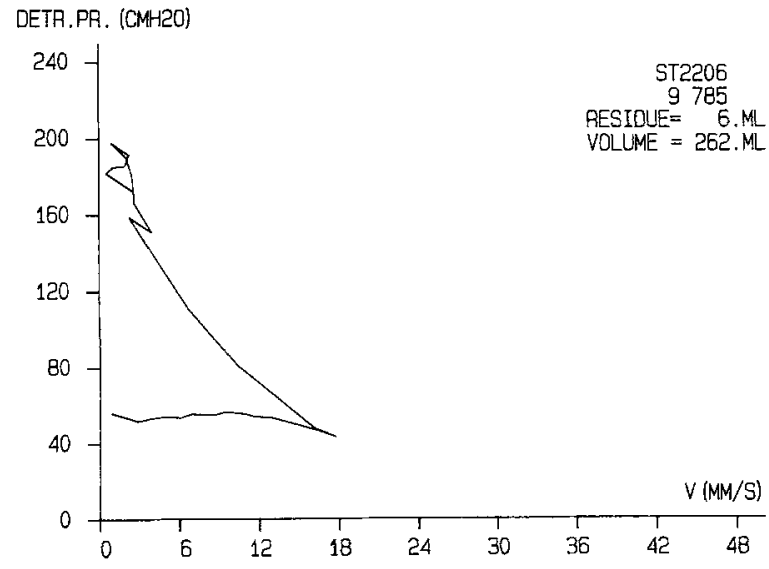

Fig. 1. Detrusor pressure as a function of circumferential shortening velocity calculated from flow rate during micturition

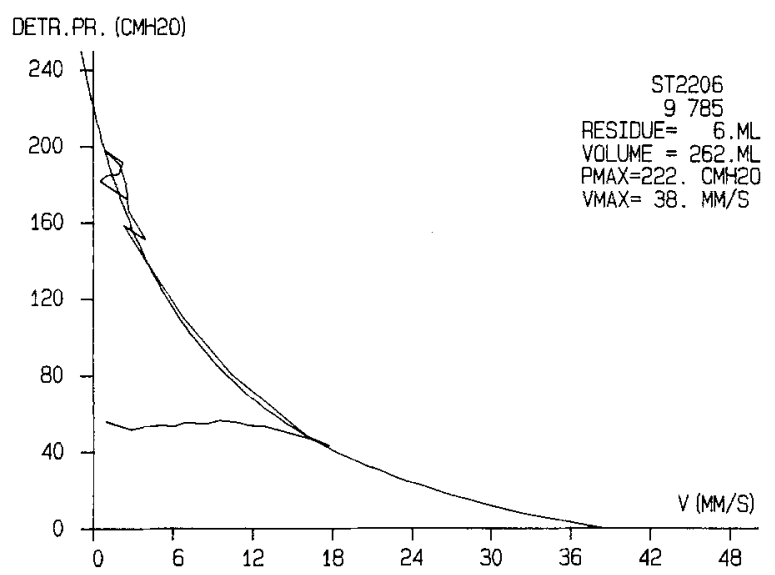

Fig. 2. Same data as in Fig. 1, fitted with a hyperbolic equation with $\mathrm{a} / \mathrm{P}_{0}=0.25, \mathrm{P}_{0}=222 \mathrm{cmH}_{2} \mathrm{O}$ and $\mathrm{v}_{\max }=38 \mathrm{~mm} / \mathrm{s}$

\section{Materials and methods}

Since 1982 flow and detrusor pressure signals from all urodynamic investigations in the Academic Hospital Rotterdam and the associated Children"s Hospital have been stored on-line in a database using the program CLIM $[8,10] .2073$ of such measurements, from a mixed group of patients, both children and adults, were reprocessed. A plot of detrusor pressure as a function of circumferential bladder wall shortening velocity (calculated from flow rate and bladder volume [7]) was presented on a graphics screen. If the plot could be fitted with a hyperbola, two points of the curve were indicated using a mouse device and a hyperbola according to equation 3 was calculated and drawn through the indicated points, yielding the parameters $P_{0}$ and $v_{\max } .241$ measurements were selected and fitted in this way. The degree of rotation of the fitted hyperbola was calculated as:

$\mathrm{c}=\mathrm{P}_{0} / \mathrm{v}_{\max }$

The relation between $\mathrm{c}$ and conventional urodynamic parameters determined from the same measurement were investigated. A relation between $c$ and the maximum flow $Q_{\max }$ was established by sorting the population of 241 measurements with respect to $Q_{\max }$ dividing it into 12 subpopulations of equal size and calculating the median values of $\mathrm{Q}_{\max }$ and $\mathrm{c}$ for each subpopulation. The resulting
12 data pairs were described by two straight lines:

$$
\text { for } \begin{aligned}
\mathrm{Q}_{\max }<10.44 \mathrm{ml} / \mathrm{s}: \mathrm{c}=8.89-0.475 * \mathrm{Q}_{\max } \\
\mathrm{Q}_{\max }>10.44 \mathrm{ml} / \mathrm{s}: \mathrm{c}=4.54-0.0583 * \mathrm{Q}_{\max }
\end{aligned}
$$

A computer program was written that calculated $c$ from the maximum flow in each measurement using Eq. 5 and calculated an estimate of $v_{\max }$, called $v_{\text {est }}$, by inserting Eq. 4 into Eq. 3 and solving for $v_{\text {max }}$. This yielded:

$\mathrm{v}_{\mathrm{est}}=\mathrm{P} /(2 \mathrm{c})+\mathrm{v} / 2+1 / 2 \sqrt{\mathrm{P}^{2} / \mathrm{c}^{2}+\mathrm{v}^{2}+18 \cdot \mathrm{P} \cdot \mathrm{v} / \mathrm{c}}$

$V_{\text {est }}$ values were calculated for all $P, v$ values throughout micturition and graphed as a function of bladder volume. Such plots were made and inspected for all 241 measurements. The maximum of each curve, $v_{\text {est }}$ max, was indicated in the curve by a pointer which could be corrected in case of artefacts. The corrected maximum, as well as the value of $v_{\text {est }}$ at the point of maximum flow $v_{\text {cst }}\left(Q_{\max }\right)$ were stored.

$\mathrm{By}$ means of multiple regression analysis possible relations between $v_{\max }$ and $P_{0}$ on the one hand and the conventional parameters $\mathrm{Q}_{\max }$ (maximum flow), $\mathrm{P}\left(\mathrm{Q}_{\max }\right.$ ) (detrusor pressure at maximum flow) and $\mathrm{Vol}$ (the bladder volume at the onset of micturition) as well as $\mathrm{P}(\max )$ (the maximum pressure attained during micturition) and $v(\max )$ (the maximum contraction velocity attained during micturition) on the other hand were investigated. Based on the results a computer program was developed that calculated an estimate for $\mathrm{P}_{0}$ (called $\mathrm{P}_{\text {est }}$ ) according to the equation:

$$
\begin{aligned}
\mathrm{P}_{\text {est }}= & 45.21+0.58 * \mathrm{P}(\max )+0.56 * \mathrm{P}\left(\mathrm{Q}_{\max }\right) \\
& +1.18 * \mathrm{v}(\max )-0.040 * \mathrm{Vol}
\end{aligned}
$$

and that fitted a hyperbola according to the relation in Eq. 3 through $\mathrm{P}_{\text {est }}$ and $\mathrm{v}(\max )$. The intersection of this hyperbola with the velocity axis was called $v_{\text {esp }}$ and was calculated in this way for the set of 241 measurements.

Possible associations between the parameters resulting from these two approaches on the one hand, and the parameters $P_{0}$ and $v_{\max }$ and the contractility parameter $w_{\max }[3,9]$ on the other hand, were tested using Spearman"s rank correlation as implemented in the statistical package SPSS. The relation between the estimated parameter $v_{\text {csp }}$ and $v_{\max }$ was expressed as a calibration factor:

$\mathrm{cal}=\mathrm{v}_{\mathrm{max}} / \mathrm{v}_{\mathrm{esp}}$

The distribution of the factor cal was calculated for the 241 measurements.

Using the urethral resistance parameter URA [4] and a discrimination value for this parameter of $28.5 \mathrm{cmH}_{2} \mathrm{O}$ [12] the 241 measurements were split into measurements from obstructed and unobstructed patients. Differences between parameter values for both groups were tested for significance using the Mann-Whitney U-test. The group of measurements from unconstructed patients was further split into micturitions with residual volume larger than or equal to $50 \mathrm{ml}$, and micturitions with residual volume less than $50 \mathrm{ml}$. Again the differences between the parameter values in the various groups were tested for significance.

\section{Results}

Figure 1 shows an example of one of the selected pressure/ velocity plots, and Fig. 2 shows the fitted hyperbola. 241 out of the 2073 pressure/velocity plots $(=12 \%)$ could be fitted in this way. There was no significant correlation between the parameters $\mathrm{P}_{0}$ and $\mathrm{v}_{\max }$. Table 1 lists correlations of the rotation $\mathrm{c}$ with other parameters determined from the same measurements, such as bladder volume 
Table 1. Spearman's rank correlation coefficients describing correlations between the rotation $c=P_{0} / v_{\max }$ of a fitted hyperbolic pressure-velocity relation and other parameters determined from the same measurements

\begin{tabular}{lcc}
\hline Correlation with & & Significance \\
\hline Volume & -0.2364 & $<0.001$ \\
$\mathrm{w}_{\max }$ & 0.1480 & 0.01 \\
$\mathrm{w}\left(\mathrm{Q}_{\max }\right)$ & -0.1606 & 0.006 \\
$\mathrm{Q}_{\max }$ & -0.3564 & $<0.001$ \\
$\mathrm{P}\left(\mathrm{Q}_{\max }\right)$ & 0.2565 & $<0.001$ \\
$\mathrm{v}\left(\mathrm{Q}_{\max }\right)$ & -0.1625 & $0.006 \quad(N=241)$ \\
\hline
\end{tabular}

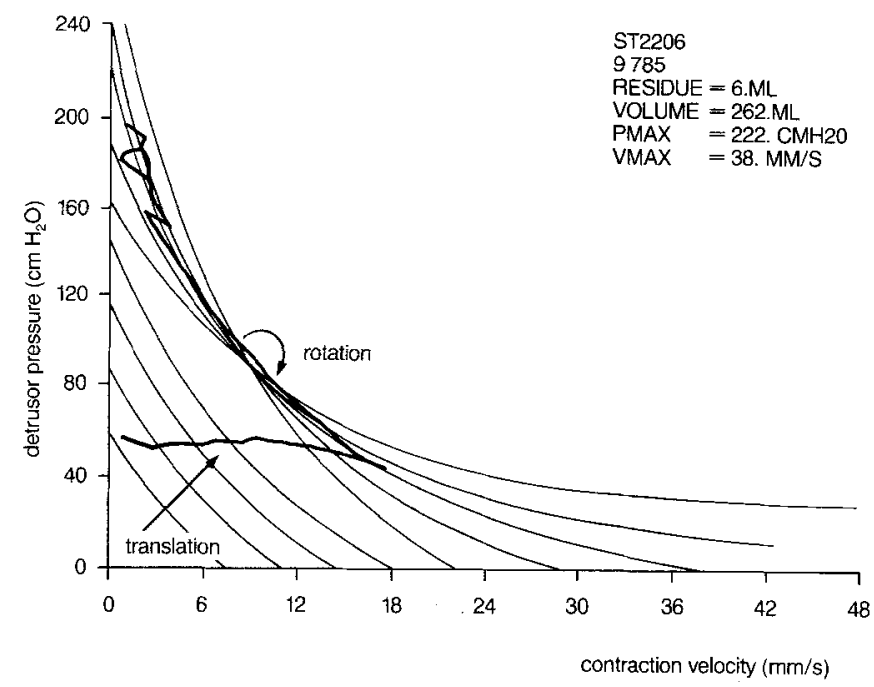

Fig. 3. Example of fitting a hyperbolic equation with preset curvature to experimental pressure-velocity data by rotation and translation of the curve

before voiding started, the maximum of the contractility parameter $w$, its value at the point of maximum flow, the maximum flowrate itself, detrusor pressure at maximum flow, and bladder contraction velocity at maximum flow. Figure 4 depicts the most significant of these correlations, the relation between $c$ and $\mathrm{Q}_{\max }$ as described by the relation in Eq. 5 . Figure 5 shows a typical example of the estimated maximum contraction velocity $v_{\text {est }}$ plotted as a function of bladder volume. The marker at approximately $200 \mathrm{ml}$ on the horizontal axis indicates the point of maximum flow. The maximum of the function, $v_{\text {est }} \max$, and its value at maximum flow, $v_{\text {est }}\left(Q_{\max }\right)$, are displayed in the upper right hand corner.

Table 2 shows the results of multiple regression analysis of $\mathrm{P}_{0}$ and $\mathrm{v}_{\text {max }}$ on the available conventional parameters and on $\mathrm{P}(\max )$ and $\mathrm{v}(\max )$, the maximum detrusor pressure and contraction velocity in each pressure-flow measurement. It can be seen that $\mathrm{P}_{0}$ can be estimated from these parameters significantly better than $\mathrm{V}_{\max }$. The table also displays the correlation between an estimator based on the regression and the original parameter as quantified by Spearman's rank correlation

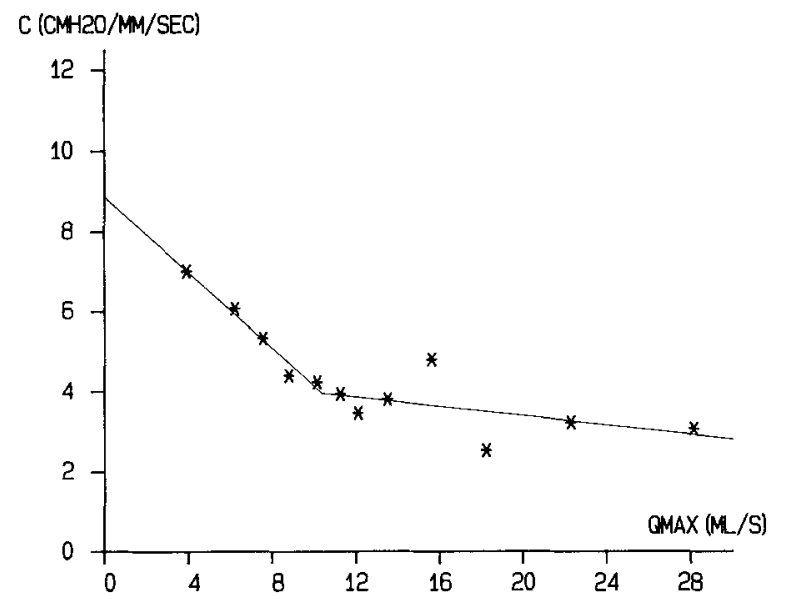

Fig. 4. Relation between the rotation $c=P_{0} / v_{\max }$ of a fitted hyperbolic pressure-velocity relation and the maximum flow $\mathrm{Q}_{\max }$. Data points indicate the median values for $\mathrm{c}$ and $\mathrm{Q}_{\max }$ calculated from 12 equally sized subgroups of 241 measurements sorted with respect to $\mathrm{Q}_{\max }$

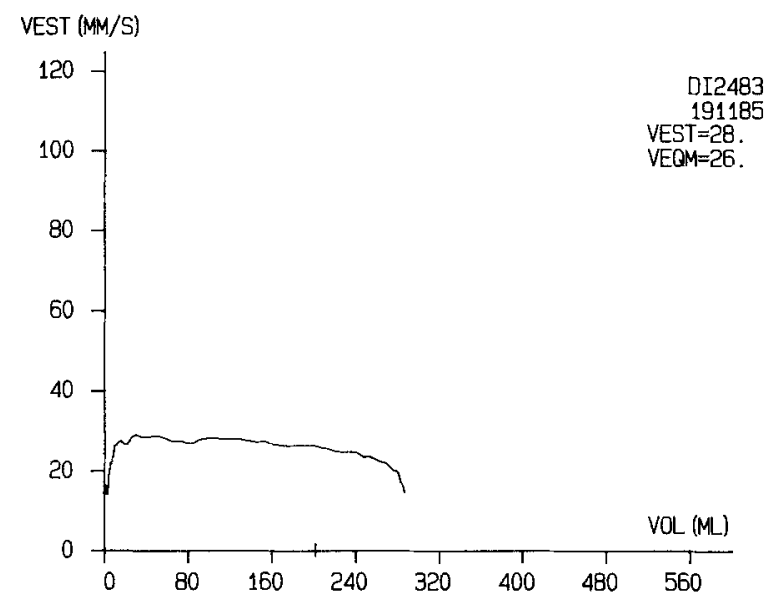

Fig. 5. Estimated contraction velocity as a function of bladder volume during emptying of the bladder. The small marker on the horizontal axis at $200 \mathrm{ml}$ indicates the point of maximum flow

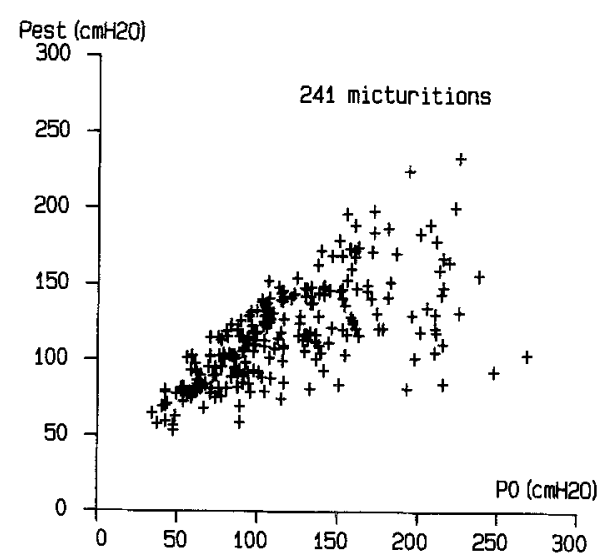

Fig. 6. Scatterplot illustrating the correlation between $P_{0}$ and $P_{\text {est }}$, an estimator for $\mathrm{P}_{0}$ based on multiple regression analysis 
Table 2. Results of multiple regression analysis of $P_{0}$ and $v_{\max }$ on conventional urodynamic parameters and $\mathrm{P}(\max )$ and $\mathrm{v}(\max )$, the maximum values attained by detrusor pressure and detrusor wall contraction velocity during micturition. The change in $\mathrm{R}$ square value caused by adding the indicated variables to the regression equation is shown. The correlation between an estimator based on the regression equation and the original variable as quantified by Spearman"s rank correlation coefficient is also shown

\begin{tabular}{llll}
\hline Regression of $\mathrm{P}_{0}$ on & \multicolumn{2}{l}{ Regression of $\mathrm{v}_{\max }$ on } \\
\hline $\mathrm{P}(\max )$ & 0.37 & $\mathrm{Q} \max$ & 0.078 \\
$\mathrm{v}(\max )$ & 0.024 & $\mathrm{P}\left(\mathrm{Q}_{\max }\right)$ & 0.15 \\
$\mathrm{P}\left(\mathrm{Q}_{\max }\right)$ & 0.032 & Volume & 0.035 \\
Volume & 0.019 & $\mathrm{v}(\max )$ & 0.0031 \\
$\mathrm{Q}_{\max }$ & 0.00008 & $\mathrm{P}(\max )$ & 0.0017 \\
\hline
\end{tabular}

Spearman's rank correlation

$0.73(N=241) \quad 0.51(N=241)$

Table 3. Correlation of estimators for $v_{\max }$ with $v_{\max }, \mathrm{P}_{0}$ and $\mathrm{w}_{\max }$. Spearman's rank correlation coefficients

\begin{tabular}{llll}
\hline Correlation with & $\mathrm{v}_{\max }$ & $\mathrm{P}_{0}$ & $\mathrm{w}_{\max }$ \\
\hline Parameter & & & \\
\hline $\mathrm{v}_{\text {est } \max }$ & & & \\
$\mathrm{v}_{\text {est }}\left(\mathrm{Q}_{\max }\right)$ & 0.56 & 0.55 & 0.84 \\
$\mathrm{v}_{\text {esp }}$ & 0.58 & 0.52 & 0.83 \\
\hline
\end{tabular}

$N=241 / 238$, all correlations are significant at the $0.1 \%$ level

coefficient. The variable $\mathrm{Q}_{\max }$ was excluded from the regression equation for $\mathrm{P}_{0}$ as it showed a minimal effect. Figure 6 is a scatterplot of the estimate for $P_{0}$ (called $P_{\text {est }}$ ) as a function of $\mathrm{P}_{0}$. Apart from a few outliers the close approximation of $\mathrm{P}_{0}$ by $\mathrm{P}_{\text {est }}$ is obvious.

Table 3 shows the correlations of the contraction velocity estimates calculated according to the first $\left(\mathrm{v}_{\text {est }} \max\right.$ and $\left.\mathrm{v}_{\mathrm{est}}\left(\mathrm{Q}_{\max }\right)\right)$ and second $\left(\mathrm{v}_{\mathrm{esp}}\right)$ method with $\mathrm{P}_{0}$, $\mathrm{V}_{\max }$ and the contractility parameter $\mathrm{w}_{\max } \cdot \mathrm{v}_{\mathrm{esp}}$ shows the highest correlation with $\mathrm{v}_{\max }$, $\mathrm{v}_{\text {est }}$ max shows a correlation

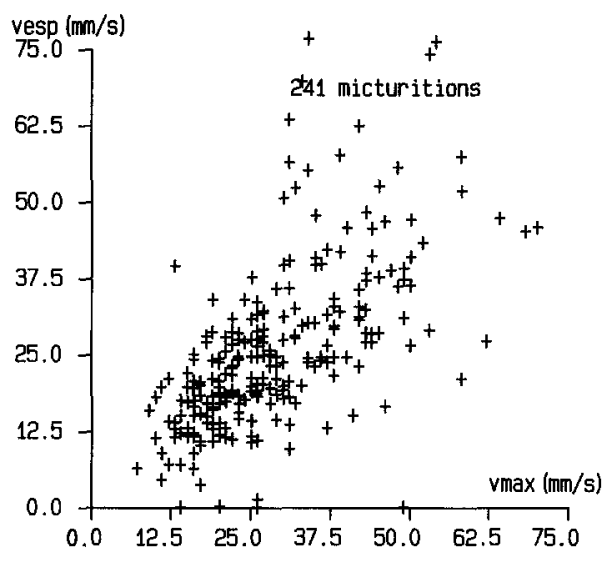

Fig. 7. Scatterplot illustrating the correlation between $v_{\max }$ and $v_{\text {esp }}$, and estimator for $v_{\max }$ based on $P_{\text {est }}$ and the point of maximum contraction velocity in a pressure-velocity plot of a complete micturition

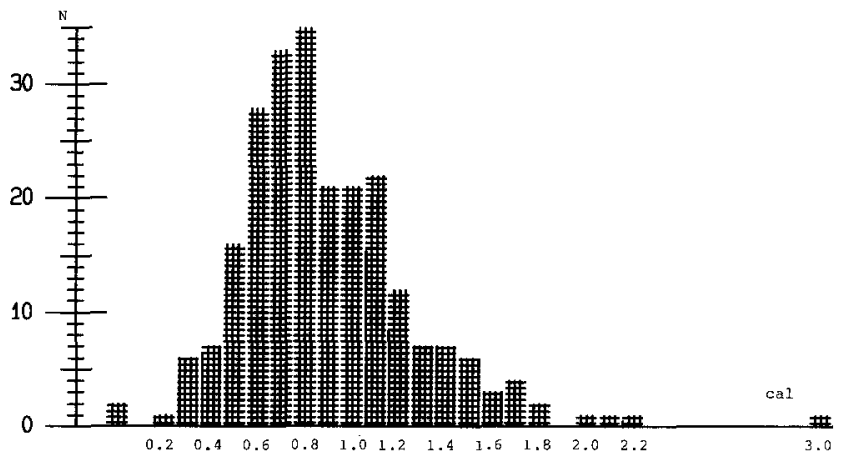

Fig. 8. Frequency distribution of the calibration factor $v_{e s p} / v_{\max }$

with $\mathrm{P}_{0}$ almost as high as that with $\mathrm{v}_{\max }$, and also the highest correlation with $\mathrm{w}_{\max }$. All correlations are significant at the $0.1 \%$ level. The relation between $\mathrm{v}_{\max }$ and $\mathrm{v}_{\mathrm{esp}}$, the "best" estimator is illustrated in Fig. 7, indicating a very good correlation apart from a few outliers. The three points at the horizontal axis represent failures of the estimation process and were excluded from further analysis. Figure 8 shows a frequency distribution of the calibration factor cal (Eq. 8). It follows that $90 \%$ of the (true) $\mathrm{v}_{\max }$ values are in the range $0.40 * \mathrm{v}_{\text {esp }}-1.40$

Table 4. Differences between parameters determined from micturitions from obstructed and unobstructed patients as seperated by the parameter URA. Standard deviations are presented in parentheses. Significance of difference was tested using Mann-Whitneys U-test

\begin{tabular}{lcccc}
\hline Parameter & Obstructed & Significance & Unobstructed & Units \\
\hline $\mathrm{P}_{0}$ & $143(39)$ & $<0.0001$ & $108(49)$ & $\mathrm{cmH}_{2} 0$ \\
$\mathrm{~V}_{\max }$ & $28.9(13.2)$ & 0.77 & $28.8(11.8)$ & $\mathrm{mm} / \mathrm{s}$ \\
$\mathrm{W}_{\max }$ & $14.5(3.8)$ & $<0.0001$ & $12.0(5.3)$ & $\mathrm{W} / \mathrm{m}^{2}$ \\
$\mathrm{~V}_{\text {est }}\left(\mathrm{Q}_{\max }\right)$ & $25.4(7.2)$ & 0.99 & $25.8(9.3)$ & $\mathrm{mm} / \mathrm{s}$ \\
$\mathrm{V}_{\text {esp }}$ & $23.9(10.7)$ & 0.31 & $27.5(15.7)$ & $\mathrm{mm} / \mathrm{s}$ \\
\hline \multicolumn{5}{c}{$N=175 / 172$} \\
\hline
\end{tabular}


Table 5. Differences between parameters determined from flow and detrusor pressure during micturition of unobstructed patients, for micturitions ending with or without significant residual urine. Standard deviations are presented in parentheses. Significance of difference was tested using Mann-Whitneys U-test

\begin{tabular}{lcccc}
\hline Parameter & Res $<50 \mathrm{ml}$ & Significance & Res $>50 \mathrm{ml}$ & Units \\
\hline $\mathrm{P}_{0}$ & $117(50)$ & $<0.0001$ & $73(24)$ & $\mathrm{cmH}_{2}$ \\
$\mathrm{~V}_{\max }$ & $30.3(12.3)$ & 0.0012 & $23.4(8.0)$ & $\mathrm{mm} / \mathrm{s}$ \\
$\mathrm{W}_{\max }$ & $13.1(5.3)$ & $<0.0001$ & $7.9(2.4)$ & $\mathrm{W} / \mathrm{m}^{2}$ \\
$\mathrm{~V}_{\text {est }}\left(\mathrm{Q}_{\max }\right)$ & $27.7(9.3)$ & $<0.0001$ & $19.0(5.2)$ & $\mathrm{mm} / \mathrm{s}$ \\
$\mathrm{V}_{\text {esp }}$ & $30.8(16.0)$ & $<0.0001$ & $15.9(5.4)$ & $\mathrm{mm} / \mathrm{s}$ \\
\hline & $N=137 / 134$ & & $N=38$ \\
\hline
\end{tabular}

* $\mathrm{v}_{\mathrm{esp}}$ so that a measure of the interval in which $\mathrm{v}_{\max }$ can be found is available. From the 241 measurements in the test population 66 fell in the category obstructed and 175 in the category non-obstructed. $v_{\max }, v_{\text {est }}\left(Q_{\max }\right)$ and $v_{\text {esp }}$ showed no significant difference between the two groups; but $\mathrm{P}_{0}$ and $\mathrm{w}_{\max }$ did show significant differences (Table 4 ). From the 175 micturitions from non-obstructed patients, 38 ended with a significant residual volume (larger than $50 \mathrm{ml}$ ) and 137 ended without such residual (Table 5). All parameters were significantly lower in the group with residual urine.

\section{Discussion}

From the tested population of 2,073 measured micturitions $12 \%$ could be fitted with a hyperbolic pressure/ velocity relation, yielding the parameters $P_{0}$ and $v_{\max }$. Two procedures for deriving estimates for the $\mathrm{v}_{\max }$ parameter which can be applied to ANY micturition, provided a valid flow and detrusor pressure signal are available, were tested. When applied to the $12 \%$ successfully fitted measurements a significant correlation between the estimated $v_{\max }$ values and the "true" values was found. Three questions may now be asked:

1. Do the estimators estimate $v_{\max }$ ?

2. Are the estimators contractility parameters?

3. Are such parameters relevant in clinical practice?

Of the proposed estimators $v_{\text {esp }}$ is more strongly correlated with $v_{\max }$ and less with $\mathrm{P}_{0}$. The calibration factor for this parameter, as depicted in Fig. 8, has a median value of 0.823 and a mode of 0.80 and the $90 \%$ interval ranges from 0.40 to 1.40 . This means that by correcting this parameter with a factor $1 / 0.8$ it would estimate the most probable value for $v_{\max }$ and "predict" a range of possible values in which the true value of the parameter would be found if it were possible to determine it.

The parameter which best estimates $v_{\max }$ is not necessarily the best contractility parameter (question 2) since $\mathrm{v}_{\max }$ might not be a contractility parameter at all. As stated before contractility is defined by the parameter pair $\left(\mathrm{P}_{0}, \mathrm{~V}_{\max }\right)$ and using only one parameter of the pair may be insufficient to describe contractility. $w_{\max }$ is a parameter which is intended to combine the information. of the parameter pair into one parameter [3]. However as the parameters $P_{0}$ and $v_{\max }$ are independent information is lost in this process. $P_{0}$ and $v_{\max }$ describe different clinically relevant aspects of the contractility of the urinary bladder. This point is illustrated in Table 4. As a first approximation no difference in contractility between measurements from obstructed and non-obstructed patients would be expected. To complicate matters, patients' bladders and thus their contractility may of course be changed secondary to the obstruction. Table 4 shows that there was no significant difference in $\mathrm{V}_{\max }$ between the two groups, and this lack of difference was faithfully reflected in the estimators $v_{\text {est }}\left(Q_{\max }\right)$ and $v_{\text {esp. }} P_{0}$ was significantly lower in the unobstructed group, and this was reflected in $w_{\max }$ (which combines $P_{0}$ and $v_{\max }$ ). Table 4 thus shows that $v_{\max }$ (and its estimators $\mathrm{v}_{\text {est2 }}\left(Q_{\max }\right)$ and $\left.\mathrm{v}_{\text {esp }}\right)$ and $\mathrm{P}_{0}$ contain different information which is combined in $w_{\max }$.

The differences between unobstructed patients voiding with and without residual urine in Table 5 must surely be due to a difference in contractility which is reflected in all the parameters listed.

It is concluded that it is possible to estimate the maximum contraction velocity of the urinary bladder from recordings of flow rate and detrusor pressure throughout micturition. The parameter $v_{\text {esp }}$ is the best estimator for this maximum contraction velocity. It is furthermore concluded that both the maximum contraction velocity and the isometric detrusor pressure of the urinary bladder represent different aspects of contractility, which are both clinically relevant.

Acknowledgement. Derek J. Griffiths performed all the urodynamic investigations on which this study is based, and did the initial processing of the data involved. He also criticized and corrected this manuscript, stimulated my thinking and was as indispensable as ever in many other ways.

\section{References}

1. Gleason DM, Bottaccini MR, Byrne JC (1987) Review of current physical and hydrodynamic concepts and their impact on urodynamics. Neurourol Urodynam 6:1

2. Griffiths DJ, van Mastrigt R (1985) The routine assessment of detrusor contraction strength. Neurourol Urodynam 4:77 
3. Griffiths DJ, Constantinous CE, van Mastright R (1986) Urinary bladder function and its control in healthy females. Am J Physiol 251:R225

4. Griffiths DJ, van Mastrigt R (1989) Quantification of urethral resistance and bladder function during voiding, with special reference to the effects of prostate size reduction on urethral obstruction due to BPH. Neurourol Urodynam 8:17

5. Hellstrand $\mathrm{P}$ (1979) Mechanical and metabolic properties related to contraction in smooth muscle. Acta Physiol Scand [Suppl] 464

6. Hill AV (1938) The heat of shortening and the dynamic constants of muscle. Proc R Soc Lond [B] 126:136

7. van Mastrigt R, Griffiths DJ (1979) Contractility of the urinary bladder. Urol Int 34:410

8. van Mastrigt R (1984) A computer program for on-line measurement, storage, analysis and retrieval of urodynamic data. Comput Prog Biomed 18:109

9. van Mastrigt R, Griffiths DJ (1986) An evaluation of contractility parameters determined from isometric contractions and micturition studies. Urol Res 14:45

10. van Mastrigt R (1987) Urodynamic analysis using an on-line computer. Neurourol Urodynam 6-3:206
11. van Mastrigt R, Griffith DJ (1987) Clinical comparison of bladder contractility parameters calculated from isometric contractions and pressure-flow studies. Urology 29:102

12. van Mastrigt $R$, Rollema $H J$ (1988) Urethral resistance and urinary bladder contractility before and after transurethral resection as determined by the computer program CLIM. Neurourol Urodynam 7:226

13. Murphy RA (1976) Contractile systgem function in mammalian smooth muscle. Blood Vessels 13:1

Dr. ir. R. van Mastrigt

Departments of Urology and

Biomedical Physics and Technology

Erasmus University Rotterdam

Room EE 1630

P.O. Box 1738

3000 DR Rotterdam

The Netherlands 